\title{
O desenvolvimento da Física de Altas Energias no Brasil: produção científica, programas de pós-graduação e grupos de pesquisa
}

\section{Development of High Energy Physics in Brazil: scientific output, graduate programs and research groups}

\section{El desarrollo de la Física de Altas Energías en Brasil: la producción científica, los programas de postgrado y los grupos de investigación}

http://dx.doi.org/10.21713/2358-2332.2016.v13.1230

Gonzalo Rubén Alvarez, mestre e doutorando no Programa de PósGraduação em Comunicação e Informação da Universidade Federal do Rio Grande do Sul (UFRGS), Porto Alegre, RS, Brasil. E-mail: gonzalorubenalvarez@gmail.com.

Samile Andréa de Souza Vanz, doutora em Comunicação e Informação, professora adjunta do Departamento de Ciências da Informação e do Programa de Pós-Graduação em Comunicação e Informação da Universidade Federal do Rio Grande do Sul (UFRGS), Porto Alegre, RS, Brasil. E-mail: samile.vanz@ufrgs.br.

\section{Resumo}

Este estudo se fundamenta em metodologia bibliométrica e analisa o desenvolvimento da Física de Altas Energias (FAE) no Brasil a partir das publicações indexadas pela Web of Science no período de 1983 a 2013. Os resultados mostram um significativo crescimento da produção científica brasileira em FAE ao longo desse intervalo. A expansão da atividade na área se associou à ampliação do número de programas de pós-graduação em Astronomia e Física com linhas de pesquisa em FAE, de professores titulados e de grupos do Conselho Nacional de Desenvolvimento Científico e Tecnológico (CNPq), à participação nas colaborações internacionais e à 
instituição da Rede Nacional de Física de Altas Energias (Renafae). Conclui que o aperfeiçoamento de políticas científicas e investimentos podem aumentar o protagonismo e a influência da ciência brasileira em FAE internacionalmente.

Palavras-chave: Produção Científica. Bibliometria. Grupos de Pesquisa. Programas de Pós-Graduação. Física de Altas Energias.

\section{Abstract}

This study is based on bibliometric methods and analyzes the development of High Energy Physics (HEP) in Brazil from papers indexed by the Web of Science from 1983 to 2013. The results show a significant growth in scientific output of Brazilian HEP over the period. The expansion of activity in the area was associated with the growth of graduate programs in Astronomy and Physics with HEP research lines, expansion of PhD professors and the National Council for Scientific and Technological Development(CNPq) research groups, participation in international collaborations, and the establishment of the Rede Nacional de Física de Altas Energias (Renafae). The conclusion is that the improvement of scientific policies and investments could increase the role and influence of Brazilian HEP internationally.

Keywords: Scientific Output. Bibliometrics. Research Groups. Graduate Programs. High Energy Physics.

\section{Resumen}

Este estudio se fundamenta en la metodología bibliométrica y analiza el desarrollo de la Física de Altas Energías (FAE) en Brasil a partir de publicaciones indexadas por la Web of Science en el período de 1983 a 2013. Los resultados muestran un significativo crecimiento de la producción científica del Brasil en FAE. La expansión de la actividad en el área fue asociada a la ampliación del número de programas de postgrado en Astronomía y Física con líneas de investigación en FAE, 
profesores titulados y grupos de investigación del Consejo Nacional de Desarrollo Científico y Tecnológico (CNPq), así como a la participación en las colaboraciones internacionales y a la institución de la Rede Nacional de Física de Altas Energías (Renafae). Se concluye que la mejoría de políticas e inversiones puede aumentar internacionalmente el protagonismo y la influencia de la ciencia brasileña en la FAE.

Palabras clave: Producción Científica. Bibliometría. Grupos de Investigación. Programas de Postgrado. Física de Altas Energías.

\section{INTRODUÇÃO}

A Física constitui, atualmente, no Brasil, uma das áreas de maior reconhecimento internacional. Esta colabora para o desenvolvimento do país por meio da geração de conhecimento científico e tecnológico. A sua consolidação começou na década de 1930 com a chegada de pesquisadores estrangeiros e com a fundação das primeiras universidades públicas em São Paulo e no Rio de Janeiro (VIEIRA; VIDEIRA, 2007). Menos de um século depois, em 2010, o conjunto de artigos brasileiros representou $2 \%$ da produção mundial da área, evidenciando um impacto superior aos artigos de nações como Japão, Rússia, Índia, China e Coreia do Sul (INSTITUTE OF PHYSICS, 2012). Em comparação com 2009, o índice de citação do ano de 2010 registrou um aumento de $26,4 \%$, segundo estudo realizado no Reino Unido pelo Institute of Physics (2012), que examinou a produção científica em Física indexada na Web of Science (WoS) no período de 2001 a 2010 entre países com reconhecida importância política e econômica (G7) e países com potencial de crescimento (BRICS).

A Física brasileira é uma área de grande visibilidade internacional, dada a quantidade considerável de artigos e citações em revistas de alto impacto (CAPES, 2013). O aumento da participação dos pesquisadores nacionais nas colaborações internacionais e a consequente intensificação das atividades científicas se tornaram pontos marcantes para o desenvolvimento da área (CAPES, 2013). Sob essa perspectiva, pode-se assegurar que seu crescimento é, em grande parte, consequência do trabalho em colaboração e da interação entre os cientistas. 
A subárea da Física que é referência no mundo em termos de colaboração é a de Altas Energias (FAE), considerada uma das disciplinas científicas mais internacionalizadas e colaborativas (MELE et al., 2006). Desde a década de 1950, grandes experimentos com aceleradores de partículas envolvem a participação de cientistas de vários países. 0 reduzido número de laboratórios, o custo operacional das pesquisas e a interdisciplinaridade são fatores que intensificam a construção de vínculos entre as nações. A supremacia de publicações de autoria múltipla em FAE é decorrência da sua forte cultura de comunicação e intercâmbio científico.

A FAE, também conhecida por Física de Partículas e Campos, é um dos subcampos da Física que contribui notoriamente para o avanço da disciplina em termos de descobertas científicas. Por meio de aceleradores, a FAE estuda as quatro interações básicas da natureza (gravitacional, eletromagnética, fraca e forte) e seus elementos fundamentais, as partículas elementares (SANTORO; NOVAES, 2003). Os estudos da área consistem, essencialmente, na observação dos resultados das colisões entre partículas. Na realização dos experimentos, são utilizados aceleradores que produzem feixes de partículas de alta energia. As descobertas da FAE serviram de fundamento teórico na busca pela compreensão da origem do universo (SBF, 1987).

Em relação aos benefícios para a sociedade, a FAE experimental contribui ativamente para áreas como a Medicina, no desenvolvimento de tomógrafos e terapia de radiação para diagnóstico e tratamento do câncer, baseada no uso de partículas subatômicas (prótons) e íons (átomos com carga positiva ou negativa); para a Tecnologia da Informação e Comunicação, na criação da world wide web como um sistema de processamento, transmissão e armazenamento de dados a partir do grande volume gerado pelos experimentos realizados com o acelerador de partículas Large Hadron Collider (LHC) da European Organization for Nuclear Research(CERN); para o Meio Ambiente e Energia, na produção de energia renovável ou "limpa" a partir do aproveitamento da grande quantidade de energia liberada pelas antipartículas e antimatéria nas suas interações; e para os processos industriais, no melhoramento da qualidade do isolamento térmico em equipamentos elétricos como geladeiras e fornos, mediante tecnologia baseada em uma película absorvente utilizada em 
aceleradores de partículas para remover pequenas quantidades de gás do interior do sistema de vácuo (SANTORO; NOVAES, 2003).

Em 2008, o Brasil começou a gestão no CERN para se tornar, sob uma nova modalidade de participação, um membro associado, pois, embora a colaboração da FAE brasileira com a organização europeia seja antiga e intensa, não há um vínculo formal estabelecido. Em número de pesquisadores, alunos e engenheiros, o Brasil figura em segundo lugar entre os países não membros com maior participação, atrás apenas do Canadá. Por outro lado, a condição de país membro associado pode outorgar às instituições brasileiras maiores benefícios em termos de participação nas pesquisas, conhecimento científico e tecnológico, publicação de trabalhos em coautoria e visibilidade internacional. Entretanto, a associação ao CERN não é suficiente para que haja um salto de qualidade na pesquisa da FAE, sendo necessário um investimento significativo na infraestrutura, na criação de novos laboratórios e na formação de recursos humanos (LÉPINE-SZILY, 2011). Ao mesmo tempo, pesquisadores brasileiros da área participam dos grandes projetos internacionais envolvendo colaborações com laboratórios como o Fermi National Accelerator Laboratory(Fermilab) e Brookhaven National Laboratory(BNL), nos Estados Unidos, e o Observatório Pierre Auger, na Argentina (CAPES, 2013).

A área da Física de Altas Energias é comumente foco de análise de pesquisadores internacionais, envolvendo aspectos sobre a produção científica, colaboração e impacto. No Brasil, existem investigações que apresentam indiretamente um panorama da FAE, como a de Vanz (2009), que constatou a formação de grandes grupos (teamworks) no campo como decorrência do alto custo da pesquisa e complexidade dos experimentos; e a de Duarte (2008), que afirmou que o desenvolvimento científico e tecnológico da FAE deve ter como base a colaboração internacional e a participação brasileira no CERN.

Com o objetivo de ampliar o conhecimento que se tem acerca da FAE brasileira e mapear sua produção científica, este estudo apresenta a análise bibliométrica dos artigos brasileiros publicados pela área no período de 1983 a 2013 e os discute a partir do resgate histórico da área, do panorama atual dos grupos de pesquisa registrados no CNPq e da evolução dos cursos de pós-graduação. A seção seguinte apresenta um 
histórico da área no Brasil e dados sobre o cenário atual dos cursos de pósgraduação. Depois, são apresentados os procedimentos metodológicos e os resultados obtidos. As considerações finais encerram o artigo.

\section{FAE BRASILEIRA: EVOLUÇÃO HISTÓRICA E CENÁRIO ATUAL}

O desenvolvimento da ciência no Brasil começou tardiamente devido à política colonial imposta por Portugal: "Para Portugal, o Brasil era menos um projeto de colonização do que um grande latifúndio a ser explorado" (SCHWARTZMAN, 2001, p. 30). O papel pioneiro estabelecido por Portugal com as navegações e a exploração marítima deu lugar a uma posição marginal entre os países europeus, com efeitos profundos sobre a herança cultural que o Brasil recebeu. No entanto, a vinda de D. João VI produziu importantes modificações na colônia: abertura dos portos à navegação e ao comercio exterior, inauguração da primeira biblioteca pública, criação de academias e escolas médicas. Alguns acontecimentos, como a concepção da Escola Politécnica de São Paulo em 1893 e da Academia Brasileira de Ciências em 1916, foram marcantes para a Física. A essas iniciativas de atividade científica se juntaram o impulso vigoroso das correntes de imigração e o crescimento industrial decorrente da Primeira Guerra Mundial (SBF, 1987).

No início da década de 1930 a Física internacional atravessava uma fase contraditória em termos de descobertas, de maneira que refletiu a instabilidade social do mundo todo, assolado pela crise econômica e política (MOTOYAMA, 2004). No Brasil, porém, a consolidação das pesquisas na área e a colaboração entre cientistas ocorreram de forma sistematizada nesse período, principalmente com a fundação das primeiras universidades e com a chegada de pesquisadores estrangeiros. Em 1934, em São Paulo, o cientista italiano Gleb Wataghin implantou o Departamento de Física na Faculdade de Filosofia, Ciências e Letras (FFCL) da Universidade de São Paulo (USP). Wataghin era, por natureza, um físico teórico; no entanto, montou um laboratório experimental e começou a estudar os raios cósmicos, a alta energia. Em 1938, a chegada de novos físicos estrangeiros à USP possibilitou o trabalho em colaboração e a realização de novas experiências com raios cósmicos. Eles introduziram no Brasil a 
tradição de Física Experimental, que vinha sendo desenvolvida por outros países (SCHWARTZMAN, 2001; MOTOYAMA, 2004). Chaves et al. (2002) reforçam a ideia de que a Física em São Paulo nasceu de fato com a FAE, experimentalmente, com os estudos sobre raios cósmicos e, teoricamente, com modelos sobre produção de partículas e teoria de campos.

Em menor escala, no Rio de Janeiro, o físico e engenheiro alemão Bernhard Gross deu início às investigações na área da Física dos Sólidos no Instituto Nacional de Tecnologia (INT) (SBF, 1987; VIEIRA; VIDEIRA, 2007). Ainda, "nesse mesmo período, Wataghin publica seu primeiro artigo no Brasil, nos anais da Academia Brasileira de Ciências, sobre propriedades de partículas elementares" (VIEIRA; VIDEIRA, 2007, p. 14).

Na década de 1940, com a consolidação do projeto universitário, os incipientes grupos de investigação experimental em Física Nuclear da USP constroem os primeiros aceleradores de partículas. "As linhas de pesquisa iniciadas no Brasil, desde então e ao longo dos anos, conseguiram se desenvolver e estabelecer parcerias com centros de pesquisa do exterior" (SBF, 2012, p. 39). Em parceria com a Fundação Rockefeller, o Departamento de Física pôde adquirir um acelerador de partículas nos Estados Unidos (SCHWARTZMAN, 2001). Enquanto isso, no Rio de Janeiro, na Faculdade Nacional de Filosofia da Universidade do Brasil, Joaquim da Costa Ribeiro continua os trabalhos iniciados por Gross no INT.

Depois da Segunda Guerra Mundial, muitos alunos de Wataghin que estavam trabalhando em laboratórios da Europa e dos Estados Unidos regressam para o Brasil em busca de novos desafios. Eles influenciam a volta de César Lattes, que tinha acabado de participar, em Bristol (Inglaterra) e em Berkeley (Estados Unidos), da descoberta do méson pi, partícula muito importante na descrição das forças nucleares. Em 1949, Joaquim da Costa Ribeiro e outros professores da Faculdade Nacional de Filosofia fundam o Centro Brasileiro de Pesquisas Físicas (CBPF) como uma unidade autônoma e privada de investigação, com o propósito de guiar o país pelo caminho da pesquisa atômica (GOLDEMBERG, 1973; SBF, 1987; SCHWARTZMAN, 2001; VIEIRA; VIDEIRA, 2007).

Motoyama (2004) destaca que o trabalho sobre a descoberta do méson pi foi recompensado com o prêmio Nobel, em 1950. O autor explica 
que o nome de Lattes não apareceu entre os ganhadores, talvez por ser de um país de pouca tradição científica, embora ele e outros pesquisadores tivessem trabalhado com o físico britânico Powell, vencedor do prêmio.

Na década de 1950, a era da industrialização recebeu destaque porque as "grandes máquinas" ou aceleradores proporcionavam a oportunidade de novas investigações no campo da Física de Partículas, principalmente em laboratórios estadunidenses - como, por exemplo, Los Alamos National Laboratory (LANL), Lawrence Berkeley National Laboratory (LBL), Brookhaven National Laboratory (BNL), e Lawrence Livermore National Laboratory (LLNL) - e europeus (a exemplo do CERN), embora Shellard (2011) expresse que nesse período existia um número insuficiente de pesquisadores e cientistas brasileiros na área. 0 autor cita também a falta de ambiente de pesquisa nas universidades, a carência de especialistas na indústria e a ausência da cultura de inovação nas empresas. A despeito de o Brasil contar com o apoio político do governo, não dispunha dos recursos financeiros e tecnológicos dos países desenvolvidos (VIEIRA; VIDEIRA, 2007).

Entretanto, o país teve singular participação na resolução de problemas teóricos e nas descobertas experimentais em nível internacional. Por causa da visão e do prestígio do almirante Álvaro Alberto e de físicos como Leite Lopes, César Lattes e Jayme Tiomno, a criação do CNPq, em 1951, estimulou o desenvolvimento da ciência brasileira e da Física em particular. No mesmo ano, criou-se a Coordenação de Aperfeiçoamento de Pessoal de Nivel Superior (Capes), outorgando importância na formação de pessoal de pós-graduação no Brasil e no exterior (SBF, 1987).

A partir da década de 1960, as investigações na área da Física passaram a se expandir para além do eixo Rio de Janeiro-São Paulo. No campo experimental, houve uma queda da atividade depois do Golpe de 1964. Com a reforma universitária em 1968, pesquisadores da primeira geração formaram novos grupos em outras capitais e estados brasileiros, como Minas Gerais, Rio Grande do Sul, Rio Grande do Norte, Paraná, Paraíba, Ceará, Bahia, Pernambuco, e Distrito Federal. Além disso, em São Paulo, foram criados grupos na cidade de São Carlos e de Campinas (na Unicamp) e, no Rio de Janeiro, na Pontifícia Universidade Católica 
(PUC-Rio). A criação de universidades federais e estaduais nessa década (e mais intensamente nas décadas posteriores) instituiu inicialmente uma demanda por físicos e, uma vez estabelecidos os grupos de trabalho, ajudou a impulsionar a formação de novos recursos humanos nessa área, destacando-se que, a partir da segunda metade da década de 1960, foi estruturado um sistema com novos PPGs no país (SBF, 1987; SCHWARTZMAN, 2001; SHELLARD, 2011; VIEIRA; VIDEIRA, 2007). De acordo com Goldemberg (1973), a partir de 1965, o Banco Nacional de Desenvolvimento Econômico (BNDES) passou a subvencionar fortemente cursos de pós-graduação e atividades de pesquisa, incluindo a compra de dois novos aceleradores nucleares para São Paulo, superando amplamente o investimento realizado pelo CNPq. Outro acontecimento importante no início da década foi a institucionalização da Fundação de Amparo à Pesquisa do Estado de São Paulo (Fapesp). "[...] a Fapesp tornou-se a principal alternativa de financiamento à pesquisa no país, ao lado das agências federais estabelecidas com finalidades semelhantes nos anos 50 e 60." (SCHWARTZMAN, 2001, p. 259).

Na década de 1970, a criação do Fundo Nacional de Desenvolvimento Científico e Tecnológico (FNDCT) e da Financiadora de Estudos e Projetos (Finep) contribuiu significativamente para o crescimento da Física no Brasil. A Física Experimental ganhou terreno a partir da maior participação em colaborações internacionais. Todavia, os anos seguintes foram marcados pela diminuição dos recursos financeiros destinados à ciência e tecnologia (C\&T). A concepção, em 1985, do Ministério de Ciência e Tecnologia (MCT) trouxe esperanças para a comunidade científica. Porém, a instabilidade econômica, no início da década de 1990, foi um obstáculo para o avanço da área (SBF, 1987; SHELLARD, 2011).

No início da década de 2000, iniciou-se o período de recuperação do FNDCT. Estruturaram-se novas redes de pesquisadores, consolidaramse as atividades das entidades estaduais de apoio à pesquisa, como a Fundação de Amparo à Pesquisa do Estado do Rio de Janeiro (Faperj), a Fundação de Amparo à Pesquisa do Estado de Minas Gerais (Fapemig), a Fundação de Amparo à Ciência e Tecnologia de Pernambuco (Facepe), a Fundação de Amparo à Pesquisa do Estado do Rio Grande do Sul (Fapergs), e houve a criação do Portal de Periódicos da Capes. 
Em 2008, o MCT instituiu a Rede Nacional de Física de Altas Energias (Renafae). A organização conta com um conselho técnicocientífico, composto por membros de diversas instituições de ensino e pesquisa do país que atuam na área. A Renafae tem como objetivos promover as investigações das propriedades das partículas e suas interações, coordenar as atividades relacionadas às grandes colaborações internacionais, e criar um programa para o desenvolvimento de instrumentação e software.

A Renafae é coordenada pelo CBPF (CBPF, 2011; SHELLARD, 2011). Além dessa designação, o CBPF está negociando no CERN o ingresso do Brasil na organização internacional em caráter de membro associado. Também em Altas Energias, a Renafae se tornou um centro regional de operação da grade internacional de computadores do maior laboratório para pesquisas do mundo (CBPF, 2011). Vinculada ao CBPF, a Renafae se estabelece como um dos princípios do programa de cooperação internacional criado pelo MCT. Entre os membros da comunidade científica da $F A E$, acredita-se que a criação da rede seja responsável pelo impulso da pesquisa brasileira nos últimos anos.

Chaves et al. (2007) consideram que a maturidade e a qualidade alcançada pela FAE brasileira refletem no número de colaborações internacionais e publicações em coautoria. Entretanto, existe pouca interação entre os pesquisadores da área e as indústrias, o que dificulta os avanços tecnológicos e freia o ritmo inovador do país. Os autores explicam também que existe uma deficiência de físicos experimentais em termos nacionais, o que poderia ser sanado a partir da adoção de medidas que incluam o melhoramento dos laboratórios de pesquisa, a criação de novos cursos de pós-graduação e a extensão do prazo de formação. No início da década de 2000, Chaves et al. (2002) comprovaram que a quantidade de físicos brasileiros experimentais de todas as áreas de pesquisa (46\%) se encontrava abaixo da fração dos países industrializados (75\%). A Física de Partículas e Campos representava 13\% do total de físicos, correspondendo 3\% somente à subárea experimental. 


\section{PROCEDIMENTOS METOdOLÓGICOS}

O corpus de análise utilizado na geração dos indicadores bibliométricos é constituído por 6.350 artigos brasileiros em FAE indexados na base multidisciplinar WoS entre 1983 e 2013. A coleta das informações e download dos registros bibliográficos foi realizada em dezembro de 2014, utilizando a opção de busca avançada (advanced search), com uso de operadores booleanos (OR, AND). Os rótulos de campo CU=País (Brazil OR Brasil) e WC=Categoria Web of Science (Physics, particles \& fields) foram utilizados na estratégia de busca para demarcação dos artigos publicados por cientistas brasileiros da FAE e para delimitação da categoria de assunto dos periódicos indexados pela WoS. Os resultados foram restritos ao tipo de documento "article", ao idioma "all languages", ao tempo estipulado de 1983 até 2013, e ao índice de citações Science Citation Index Expanded (SCl-Expanded).

Na caracterização da FAE brasileira, foram utilizados indicadores bibliométricos gerais e específicos. Indicadores de produção: Publicação Total (PT), Taxa de Crescimento (TC), Publicações Acumuladas por Ano (PAA), Crescimento Acumulado por Ano (CAA), Variações por Ano (VA). Indicadores de colaboração: Colaboração Internacional (COI), Colaboração Nacional (CON), Sem Colaboração (SemC). Na geração dos indicadores de colaboração, os nomes das instituições e países foram padronizados por meio da lista de autoridades do Grupo de Pesquisa em Comunicação Científica da Universidade Federal do Rio Grande do Sul (UFRGS). Os softwares utilizados no tratamento dos dados foram BibExcel e R (análises descritivas) e Microsoft Excel 2007.

O Diretório dos Grupos de Pesquisa do CNPq (DGP/CNPq) forneceu informações acerca dos grupos cadastrados da FAE e suas áreas de atuação. A consulta parametrizada à base de dados corrente e a coleta das informações ocorreu em 24 de janeiro de 2015, aplicando a busca nos campos: nome do grupo, nome da linha de pesquisa e palavrachave da linha de pesquisa. Foram utilizadas os termos "Física de Altas Energias" e "Física de Partículas e Campos". 
O sistema de avaliação implantado pela Capes proporcionou dados quantitativos a respeito dos programas brasileiros em Física e Astronomia com linhas de pesquisa em FAE que integram o Sistema Nacional de PósGraduação (SNPG, 2015). A consulta e coleta das informações ocorreu em 26 de janeiro de 2015. Ressalta-se que não foram localizados dados sobre programas específicos em Física de Altas Energias.

\section{RESULTADOS}

A produção científica da FAE brasileira indexada na WoS no período de 1983 a 2013 é constituída por 6.350 artigos, representando $3 \%$ do total da pesquisa mundial na área. Em termos de participação, o Brasil ocupa o $14^{\circ}$ lugar no ranking de países mais produtivos, sendo que os Estados Unidos (29,78\%) lideram a lista de nações. Por outro lado, a média anual de crescimento das publicações brasileiras $(14,27 \%$ ) é amplamente superior à do mundo (3,78\%). Observa-se que a FAE nacional apresentou um crescimento mais irregular quando comparado ao do mundo, destacando que houve uma aceleração da produção entre 2009 e 2013, período posterior à criação da Renafae (Tabela 1). A ampliação do número de periódicos nacionais indexados na WoS entre 2007 e 2008 e o decorrente incremento verificado na produção científica do Brasil (LETA, 2012) não afetou a $F A E$, uma vez que se observou uma taxa de crescimento negativa em $2008(-18,91 \%)$. A FAE nacional demonstrou um crescimento ininterrupto do número de publicações nos anos subsequentes. A criação de novos grupos de pesquisa do CNPq, a consolidação e ampliação dos PPGs, o acréscimo de recursos financeiros e o papel articulador fundamental da Renafae para as colaborações brasileiras nos experimentos internacionais podem ter sido fatores determinantes para o incremento do volume de trabalhos nesses anos e, consequentemente, para o desenvolvimento da área. 
Tabela 1 - Evolução anual da atividade científica e colaboração da FAE brasileira (1983-2013)

\begin{tabular}{|c|c|c|c|c|c|c|c|c|}
\hline \multirow{2}{*}{ Ano } & \multicolumn{5}{|c|}{ Produção } & \multicolumn{3}{|c|}{ \% Colaboração } \\
\hline & PT & TC & PAA & CAA $(\%)$ & VA & COI & CON & SemC \\
\hline 1983 & 25 & .. & 25 & 0,39 & 25 & 22,22 & 0,00 & 77,78 \\
\hline 1984 & 32 & 28,00 & 57 & 0,89 & 7 & 13,33 & 20,00 & 66,67 \\
\hline 1985 & 30 & $-6,25$ & 87 & 1,36 & -2 & 18,75 & 18,75 & 62,50 \\
\hline 1986 & 47 & 56,66 & 134 & 2,10 & 17 & 37,50 & 12,50 & 50,00 \\
\hline 1987 & 39 & $-17,02$ & 173 & 2,71 & -8 & 27,27 & 9,10 & 63,63 \\
\hline 1988 & 44 & 12,82 & 217 & 3,40 & 5 & 47,37 & 5,26 & 47,37 \\
\hline 1989 & 93 & 111,36 & 310 & 4,86 & 49 & 26,53 & 10,20 & 63,27 \\
\hline 1990 & 54 & $-41,93$ & 364 & 5,71 & -39 & 37,93 & 24,14 & 37,93 \\
\hline 1991 & 73 & 35,18 & 437 & 6,86 & 19 & 37,78 & 2,22 & 60,00 \\
\hline 1992 & 112 & 53,42 & 549 & 8,62 & 39 & 53,73 & 2,99 & 43,28 \\
\hline 1993 & 97 & $-13,39$ & 646 & 10,15 & -15 & 31,67 & 6,67 & 61,67 \\
\hline 1994 & 123 & 26,80 & 769 & 12,09 & 26 & 45,35 & 10,47 & 44,19 \\
\hline 1995 & 135 & 9,75 & 904 & 14,22 & 12 & 52,43 & 8,74 & 38,83 \\
\hline 1996 & 153 & 13,33 & 1.057 & 16,63 & 18 & 48,54 & 9,71 & 41,75 \\
\hline 1997 & 190 & 24,18 & 1.247 & 19,62 & 37 & 43,38 & 6,62 & 50,00 \\
\hline 1998 & 221 & 16,31 & 1.468 & 23,10 & 31 & 54,75 & 13,12 & 32,13 \\
\hline 1999 & 251 & 13,57 & 1.719 & 27,05 & 30 & 45,02 & 17,53 & 37,45 \\
\hline 2000 & 231 & $-7,96$ & 1.950 & 30,69 & -20 & 47,19 & 21,21 & 31,60 \\
\hline 2001 & 244 & 5,62 & 2.194 & 34,53 & 13 & 46,72 & 17,21 & 36,07 \\
\hline 2002 & 308 & 26,22 & 2.502 & 39,38 & 64 & 44,16 & 19,48 & 36,36 \\
\hline 2003 & 265 & $-13,96$ & 2.767 & 43,55 & -43 & 45,28 & 24,53 & 30,19 \\
\hline 2004 & 300 & 13,20 & 3.067 & 48,27 & 35 & 38,33 & 25,67 & 36,00 \\
\hline 2005 & 260 & $-13,33$ & 3.327 & 52,36 & -40 & 45,00 & 24,62 & 30,38 \\
\hline 2006 & 274 & 5,38 & 3.601 & 56,67 & 14 & 44,89 & 22,99 & 32,12 \\
\hline 2007 & 349 & 27,37 & 3.950 & 62,17 & 75 & 37,82 & 26,65 & 35,53 \\
\hline 2008 & 283 & $-18,91$ & 4.233 & 66,63 & -66 & 40,28 & 28,62 & 31,10 \\
\hline 2009 & 271 & $-4,24$ & 4.504 & 70,90 & -12 & 45,02 & 23,25 & 31,73 \\
\hline 2010 & 309 & 14,02 & 4.813 & 75,77 & 38 & 46,93 & 22,98 & 30,10 \\
\hline 2011 & 448 & 44,98 & 5.261 & 82,83 & 139 & 57,14 & 20,54 & 22,32 \\
\hline 2012 & 512 & 14,28 & 5.773 & 90,89 & 64 & 65,23 & 17,19 & 17,58 \\
\hline 2013 & 577 & 12,69 & 6.350 & 100,00 & 65 & 67,24 & 14,04 & 18,72 \\
\hline Total & 6.350 & 14,27 & .. & .. & 18,61 & 49,07 & 19,19 & 31,74 \\
\hline
\end{tabular}

Fonte: Web of Science.

Legenda: Publicação Total (PT), Taxa de Crescimento (TC), Publicações Acumuladas por Ano (PAA), Crescimento Acumulado por Ano (CAA), Variações por Ano (VA), Colaboração Internacional (COI), Colaboração Nacional (CON), Sem Colaboração (SemC). 
As taxas de crescimento mais elevadas da produção científica da FAE se registraram em 1986 (56,66\%), $1989(111,36 \%)$ e 1992 (53,42\%). Por outro lado, identificaram-se nove taxas com valores negativos: três de 1983 a 1992, duas de 1993 a 2002 e quatro de 2003 a 2013.0 número de casos com valores negativos no período analisado não interferiu no crescimento da pesquisa em $F A E$, visto que a média anual da taxa foi de $14,27 \%$. A média anual da taxa se mostra superior aos $10,7 \%$ exibidos por Almeida e Guimarães (2013) para a produção científica brasileira no período de 1980 a 2010; superior aos 6,40\% e 6,76\% encontrados por Calero (2009) para a produção científica da Física de Partículas espanhola indexada no SCl e no arXiv entre 2000 e 2005; e superior aos 12,5\% revelados por Vanz (2009) para os artigos da Física brasileira publicados entre 2004 e 2006.

Os acréscimos e decréscimos (variações) da produção científica entre anos contínuos mostraram uma taxa média de crescimento de 18,61 trabalhos por ano (Tabela 1). A média se mostra superior aos 9,7 apresentados por Collazo-Reyes e Luna-Morales (2002) para os trabalhos publicados pelos cientistas mexicanos da Física de Partículas no período de 1971 a 2000. 0 período de 2003 a 2013 apresentou o maior número de decréscimos por ano (2003, 2005, 2008, 2009).

No que se refere à investigação da colaboração entre instituições na FAE brasileira no período de 1983 a 2013, o índice de colaboração internacional da área $(49,07 \%)$ é superior aos 30\% verificados na produção científica brasileira em anos recentes (VANZ; STUMPF, 2012). Em contraposição, o índice de colaboração nacional é acentuadamente inferior $(19,19 \%)$. Nota-se que $31,74 \%$ dos artigos foram escritos por uma única instituição (Tabela 1). A taxa é levemente inferior aos 33,90\% exibidos por Vanz (2009) para os artigos da Física brasileira indexados no SCl entre 2004 e 2006. A diminuição percentual do número de papers sem colaboração e em colaboração nacional a partir de 2009 se contrapõe ao aumento do número de artigos em colaboração internacional, inferindo-se uma maior inserção das instituições brasileiras nos grandes projetos multinacionais da área no último quinquênio.

O crescimento do volume de publicações da FAE brasileira se tornou mais notório quando o produto resultante da atividade científica 
foi agrupado em períodos de dez anos (1983-1992, 1993-2002, 2003$2013^{1}$ ), conforme Figura 1. 0 primeiro representa 8,62\% (549 artigos), o segundo, 30,76\% (1.953 artigos), e o terceiro, 60,62\% (3.848 artigos) da produção total. Constatou-se um marcante aumento do número de publicações no período de 1993 a 2002, visto que a taxa de crescimento com relação ao período anterior foi de $255,73 \%$. Embora o percentual de crescimento tenha diminuído para 97,03\% em 2003-2013, a produção quase duplicou no último período (Figura 1). Apesar de as diferenças verificadas no quantitativo de artigos publicados em cada decênio, a produção científica da FAE brasileira se manteve crescente no período.

\section{Figura 1 - Número de artigos da FAE brasileira por série temporal e taxa de crescimento (1983-2013)}

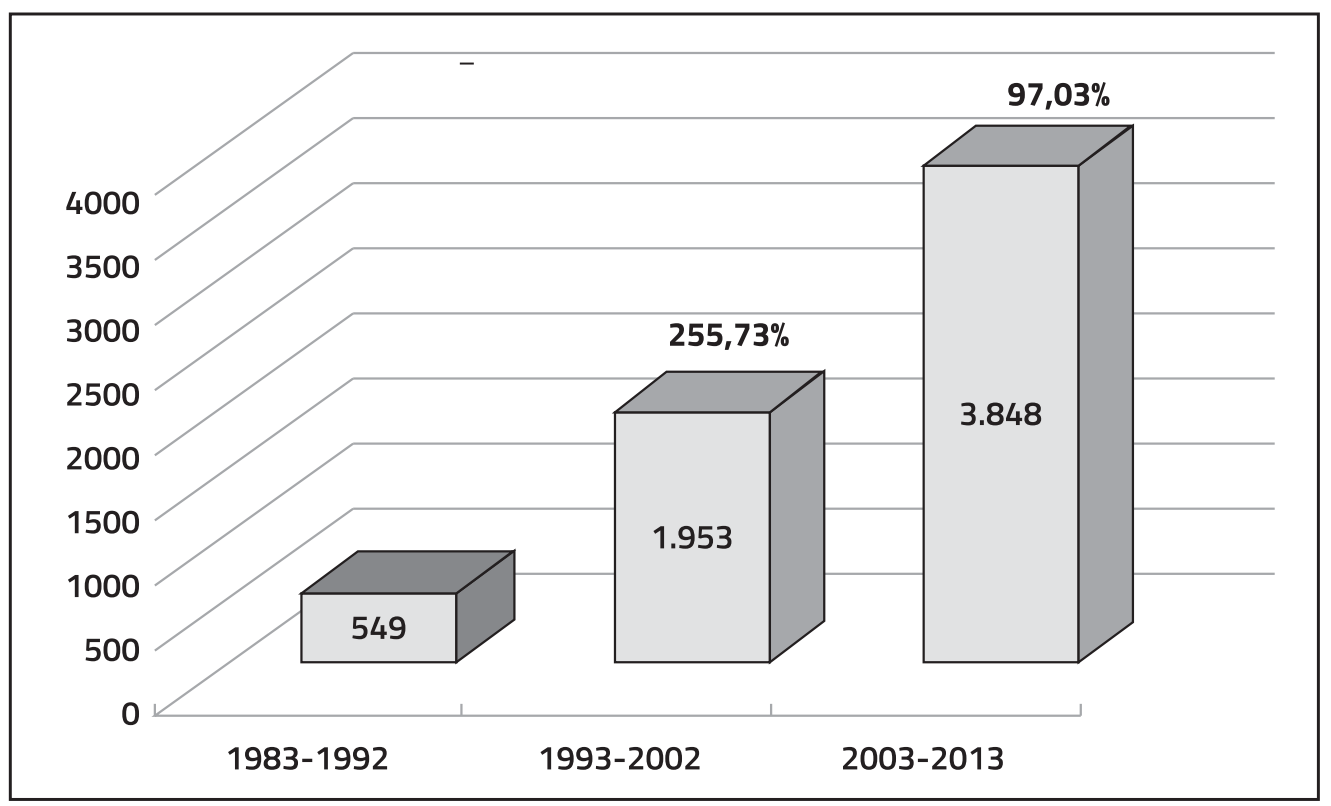

Fonte: Web of Science.

Do mesmo modo, foi possível também verificar a dispersão das publicações da área: 40\% nos primeiros vinte anos (1983-2002) e 60\% nos últimos dez (2003-2013). O crescimento de cada uma das séries temporais analisadas foi ajustado a diferentes modelos de regressão, sendo possível avaliar o nível de correlação entre as variáveis "artigos" e "ano de publicação" (Figura 2). Verificou-se que o crescimento da produção científica no período de 1983-1992 se ajustou melhor ao modelo de regressão exponencial, com um coeficiente de determinação $\left(R^{2}\right)$ igual a 0,8174 . Por outro lado, o crescimento da produção científica nos períodos 
de 1993-2002 e 2003-2013 se adequou melhor ao modelo de regressão polinomial, com um coeficiente de determinação $\left(R^{2}\right)$ igual a 0,9452 e 0,8728, respectivamente. Os três períodos apresentaram dinâmicas de produção diferentes; no entanto, exibiram taxas de crescimento com valores positivos. Na Figura 2, aprecia-se a dinâmica do crescimento da produção científica da FAE brasileira por períodos, ajustada às linhas de tendência exponencial (1983-1992) e polinomial (1993-2002; 20032013).

Figura 2 - Dinâmica de crescimento da produção científica da FAE brasileira por períodos (1983-2013)

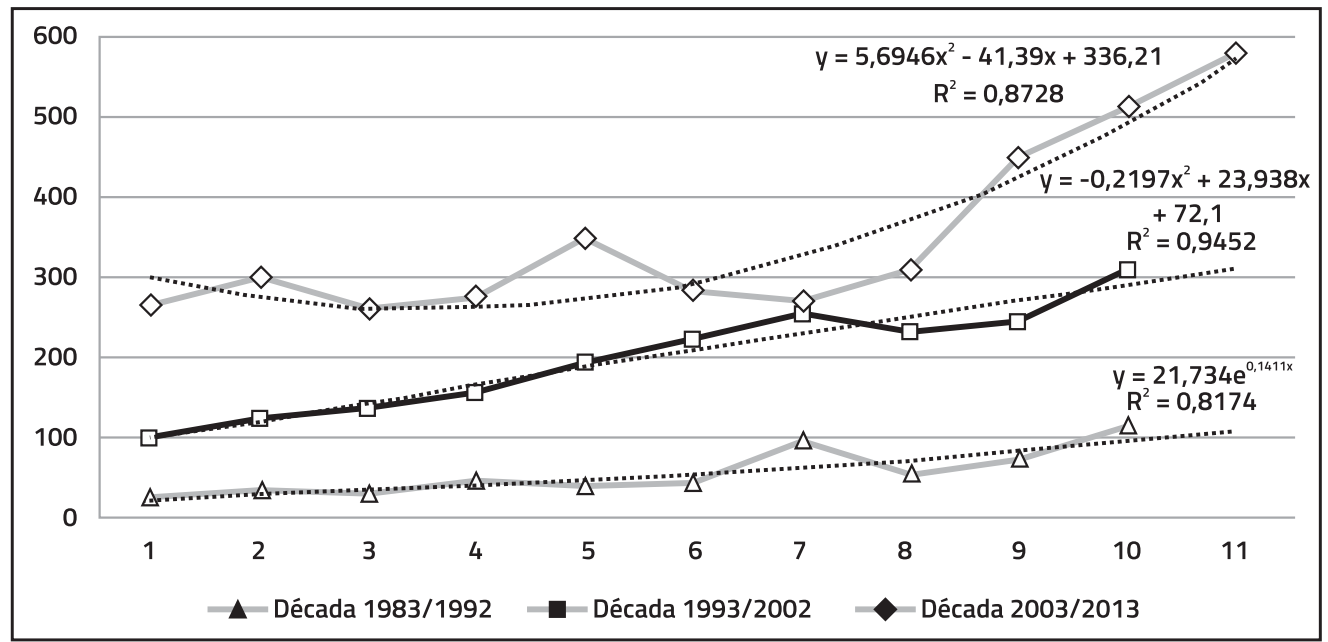

Fonte: Web of Science.

Quanto à evolução da pesquisa, o terceiro período da FAE brasileira (2003-2013) mostra uma aceleração no crescimento da produção científica, coincidindo com os resultados obtidos por Collazo-Reyes e LunaMorales (2002) para o terceiro período da Física de Partículas mexicana (1991-2000). Entretanto, a quantidade de trabalhos acumulados no Brasil em FAE é menos representativa nessa série temporal, 60,62\% contra 75\% da produção total do México em Física de Partículas.

A respeito da atividade científica da FAE brasileira, realizou-se uma busca no DGP/CNPq a fim de distinguir os grupos de pesquisa cadastrados e suas áreas de atuação. De tal modo, examinou-se, no diretório por nome do grupo, nome e palavra-chave da linha de pesquisa, sendo utilizadas as expressões: "Física de Altas Energias" e "Física de Partículas e Campos". 
Na Tabela 2, aprecia-se a distribuição dos grupos de pesquisa da FAE brasileira cadastrados no DGP/CNPq por área do conhecimento. Identificaram-se 28 grupos, dispostos da seguinte forma: 23 na área de Física, dois na Engenharia Elétrica, dois na Engenharia Nuclear e um na Matemática. Os campos mencionados se encontram inseridos na grande área das Ciências Exatas. Observou-se pouca dispersão da FAE nas diferentes áreas do conhecimento. Embora o campo estudado tenha um viés multidisciplinar, uma vez que várias ciências contribuem para o seu desenvolvimento, constatou-se que a maior parte da pesquisa científica acontece dentro da área da Física.

\section{Tabela 2 - Distribuição dos grupos de pesquisa do CNPq da FAE brasileira por área}

\begin{tabular}{|c|c|c|c|}
\hline Área & Nº de grupos & $\%$ & $\sum \%$ \\
\hline Física & 23 & 82,15 & 82,15 \\
\hline Engenharia Elétrica & 2 & 7,15 & 89,30 \\
\hline Engenharia Nuclear & 2 & 7,15 & 96,45 \\
\hline Matemática & 1 & 3,55 & 100,00 \\
\hline Total & 28 & 100,00 & .. \\
\hline
\end{tabular}

Fonte: DGP/CNPq.

O desenvolvimento da produção científica da FAE brasileira também vai ao encontro do aumento do número de grupos de pesquisa do CNPq, observado no período de 1983 a 2013. Apesar de existirem PPGs com linhas de investigação desde a década de 1960, constatou-se que, no início de 1980, a quantidade de grupos constituídos ainda era pequena, visto que até 1983 se identificaram no DGP/CNPq apenas dois grupos: Laboratório de Instrumentação e Partículas da USP (1972) e Grupo de Física de Altas Energias da Unicamp (1980). Presume-se que o aumento da colaboração científica, o maior financiamento, a criação do MCT e os avanços tecnológicos foram fatores decisivos para o incremento de novos grupos, sendo que no período de 1983 a 1992 foram formados mais quatro grupos.

Percebeu-se que, no período de 1993 a 2002, houve uma ampliação de instituições brasileiras envolvidas com a pesquisa em FAE. Nessa série temporal se instituíram sete grupos, representando 25,92\% 
do total localizado no DGP/CNPq. Vieira e Videira (2011) explicam que apesar de a mudança na organização da pesquisa científica e do aumento da colaboração, no Brasil e em outros países periféricos, a Física, até a segunda metade da década de 1990, foi feita geralmente em pequenos grupos.

No período de 2003 a 2013, comprovou-se um crescimento significativo de instituições, principalmente universidades públicas, uma vez que o número de grupos duplicou com relação à série temporal anterior, representando mais da metade da totalidade encontrada no DGP/CNPq, o qual representa 51,85\%. Notou-se que o maior incremento aconteceu no período de 2008 a 2013, quando foram criadas nove instituições. Entende-se que o setor mais produtivo da FAE brasileira é a universidade, coincidindo com os resultados de Calero (2009) para a produção científica espanhola de Física indexada no SCI no período de 2000 a 2005. Os 28 grupos de pesquisa da FAE brasileira no CNPq se encontram distribuídos em 20 instituições, sendo 18 de ensino superior e dois centros de pesquisa (Tabela 3).

Tabela 3 - Distribuição dos grupos de pesquisa do CNPq da FAE brasileira por instituição

\begin{tabular}{l|c|c|c}
\multicolumn{1}{c|}{ Instituição } & Região & $\begin{array}{c}\text { Grupos do } \\
\text { CNPq }\end{array}$ & $\%$ \\
\hline Univ. Fed. do Rio de Janeiro (UFRJ) & Sudeste & 3 & 10,75 \\
\hline $\begin{array}{l}\text { Centro Brasileiro de Pesquisas Físicas } \\
\text { (CBPF) }\end{array}$ & Sudeste & 2 & 7,14 \\
\hline $\begin{array}{l}\text { Centro Federal de Educação Tecnológica } \\
\text { Celso Suckow da Fonseca (Cefet-RJ) }\end{array}$ & Sudeste & 2 & 7,14 \\
\hline $\begin{array}{l}\text { Comissão Nacional de Energia Nuclear } \\
\text { (CNEN) }\end{array}$ & Sudeste & 2 & 7,14 \\
\hline Univ. Fed. de Goiás (UFG) & Centro-Oeste & 2 & 7,14 \\
\hline Univ. Estadual Paulista (Unesp) & Sudeste & 2 & 7,14 \\
\hline Univ. Estadual de Campinas (Unicamp) & Sudeste & 2 & 7,14 \\
\hline Instituto Federal Fluminense (IFF) & Sudeste & 1 & 3,57 \\
\hline Instituto Federal Farroupilha (IFFar) & Sul & 1 & 3,57 \\
\hline $\begin{array}{l}\text { Pont. Univ. Católica do Rio de Janeiro } \\
\text { (PUC-Rio) }\end{array}$ & Sudeste & 1 & 3,57 \\
\hline Univ. do Estado do Rio de Janeiro (UERJ) & Sudeste & 1 & 3,57 \\
\hline Univ. Fed. do ABC (UFABC) & Sudeste & 1 & 3,57 \\
\hline
\end{tabular}




\begin{tabular}{l|c|c|c}
\hline \multicolumn{1}{c|}{ Instituição } & Região & $\begin{array}{c}\text { Grupos do } \\
\text { CNPq }\end{array}$ & $\%$ \\
\hline Univ. Fed. do Espírito Santo (UFES) & Sudeste & 1 & 3,57 \\
\hline Univ. Fed. do Maranhão (UFMA) & Nordeste & 1 & 3,57 \\
\hline Univ. Fed. do Rio Grande do Sul (UFRGS) & Sul & 1 & 3,57 \\
\hline Univ. Fed. do Rio Grande do Norte (UFRN) & Nordeste & 1 & 3,57 \\
\hline Univ. Fed. de Sergipe (UFS) & Nordeste & 1 & 3,57 \\
\hline Univ. de Brasília (UnB) & Centro-Oeste & 1 & 3,57 \\
\hline Univ. Fed. de São Paulo (Unifesp) & Sudeste & 1 & 3,57 \\
\hline Univ. de São Paulo (USP) & Sudeste & 1 & 3,57 \\
\hline Total & $\mathbf{~ . .}$ & $\mathbf{2 8}$ & $\mathbf{1 0 0 , 0 0}$ \\
\hline
\end{tabular}

Fonte: DGP/CNPq.

Segundo a avaliação trienal $2013^{2}$ da Capes no Brasil, existem 58 programas de pós-graduação na área de Física e Astronomia. A distribuição dos programas por região mostra a supremacia do Sudeste (28 PPGs). Em seguida, destacam-se o Nordeste com 13, o Sul com 11, e o Centro-Oeste e o Norte com três PPGs cada um (CAPES, 2013). No Quadro 1 relacionam-se somente aqueles PPGs em Física e Astronomia cujos campos de investigação estão vinculados à FAE brasileira.

\section{Quadro 1 - PPGs brasileiros em Física e Astronomia com linhas de pesquisa em FAE}

\begin{tabular}{|c|c|c|c|c|c|c|}
\hline \multirow{2}{*}{ Instituição } & \multirow{2}{*}{ Programa } & \multirow{2}{*}{$\begin{array}{l}\text { Linha de } \\
\text { pesquisa em } \\
\text { FAE }\end{array}$} & \multicolumn{2}{|c|}{$\begin{array}{c}\text { Ano de início } \\
\text { [2] }\end{array}$} & \multirow{2}{*}{$\begin{array}{c}\text { Avaliação } \\
\text { trienal } \\
2013\end{array}$} & \multirow{2}{*}{$\begin{array}{c}\text { Docentes } \\
\text { permanentes } \\
2010-12 \text { [3] }\end{array}$} \\
\hline & & & M & D & & \\
\hline CBPF & Física & $\begin{array}{l}\text { Altas Energias; } \\
\text { Teoria de } \\
\text { Campos }\end{array}$ & 1962 & 1962 & 7 & 48 \\
\hline CBPF & Física [1] & $\begin{array}{l}\text { Detecção de } \\
\text { Partículas }\end{array}$ & 1999 & .. & 4 & 20 \\
\hline FURG & Física & $\begin{array}{l}\text { Partículas, } \\
\text { Campos E } \\
\text { Astrofísica }\end{array}$ & 2010 & .. & 3 & 14 \\
\hline IFT/Unesp & Física & $\begin{array}{l}\text { Exp. De Altas } \\
\text { Energias; } \\
\text { Partículas }\end{array}$ & 1971 & 1971 & 7 & 21 \\
\hline INPE & Astrofísica & $\begin{array}{l}\text { Astrofísica de } \\
\text { Altas Energias }\end{array}$ & 1994 & 1994 & 4 & 13 \\
\hline
\end{tabular}

2 Triênio avaliado: 2010- 


\begin{tabular}{|c|c|c|c|c|c|c|}
\hline \multirow{2}{*}{ Instituição } & \multirow{2}{*}{ Programa } & \multirow{2}{*}{$\begin{array}{l}\text { Linha de } \\
\text { pesquisa em } \\
\text { FAE }\end{array}$} & \multicolumn{2}{|c|}{$\begin{array}{c}\text { Ano de início } \\
\text { [2] }\end{array}$} & \multirow{2}{*}{$\begin{array}{c}\text { Avaliação } \\
\text { trienal } \\
2013\end{array}$} & \multirow{2}{*}{$\begin{array}{c}\text { Docentes } \\
\text { permanentes } \\
\text { 2010-12 [3] }\end{array}$} \\
\hline & & & $\mathbf{M}$ & D & & \\
\hline PUC-Rio & Física & $\begin{array}{l}\text { Fenomenologia } \\
\text { de Partículas }\end{array}$ & 1965 & 1968 & 6 & 17 \\
\hline UEL & Física & $\begin{array}{l}\text { Partículas e } \\
\text { Campos }\end{array}$ & 1996 & 2009 & 4 & 12 \\
\hline UERJ & Física & $\begin{array}{l}\text { Exp. de Altas } \\
\text { Energias; } \\
\text { Teoria de } \\
\text { Campos }\end{array}$ & 1997 & 2003 & 5 & 26 \\
\hline UFABC & Física & $\begin{array}{l}\text { Partículas e } \\
\text { Campos }\end{array}$ & 2007 & 2009 & 5 & 35 \\
\hline UFAL & $\begin{array}{l}\text { Física da } \\
\text { matéria } \\
\text { condensada }\end{array}$ & $\begin{array}{l}\text { Partículas e } \\
\text { Campos }\end{array}$ & 1992 & 1999 & 5 & 18 \\
\hline UFCG & Física & $\begin{array}{l}\text { Partículas, } \\
\text { Cosmologia e } \\
\text { Gravitação }\end{array}$ & 2007 &.. & 3 & 17 \\
\hline UFES & Física & $\begin{array}{l}\text { Interações } \\
\text { Fundamentais }\end{array}$ & 1992 & 2003 & 4 & 15 \\
\hline UFF & Física & $\begin{array}{l}\text { Exp. de Altas } \\
\text { Energias }\end{array}$ & 1977 & 1985 & 6 & 54 \\
\hline UFG & Física & $\begin{array}{l}\text { Partículas e } \\
\text { Campos }\end{array}$ & 1992 & 2008 & 4 & 22 \\
\hline UFJF & Física & $\begin{array}{l}\text { Teoria de } \\
\text { Campos }\end{array}$ & 1999 & .. & 4 & 17 \\
\hline UFMA & Física & $\begin{array}{l}\text { Teoria de } \\
\text { Campos e } \\
\text { Gravitação } \\
\end{array}$ & 2005 & 2011 & 4 & 12 \\
\hline UFMG & Física & $\begin{array}{l}\text { Teoria de } \\
\text { Campos }\end{array}$ & 1968 & 1974 & 7 & 61 \\
\hline UFPB/JP & Física & $\begin{array}{l}\text { Partículas } \\
\text { Elementares } \\
\text { e Teoria de } \\
\text { Campos }\end{array}$ & 1973 & 1980 & 5 & 18 \\
\hline UFPE & Física & $\begin{array}{l}\text { Teoria de } \\
\text { Campos }\end{array}$ & 1973 & 1975 & 6 & 37 \\
\hline UFPEL & Física & $\begin{array}{l}\text { Física ee } \\
\text { Partículas e } \\
\text { Hádrons }\end{array}$ & 2008 & .. & 4 & 11 \\
\hline UFRGS & Física & $\begin{array}{l}\text { Fenomenologia } \\
\text { de Partículas }\end{array}$ & 1964 & 1968 & 7 & 54 \\
\hline
\end{tabular}




\begin{tabular}{|c|c|c|c|c|c|c|}
\hline \multirow{2}{*}{ Instituição } & \multirow{2}{*}{ Programa } & \multirow{2}{*}{$\begin{array}{c}\text { Linha de } \\
\text { pesquisa em } \\
\text { FAE }\end{array}$} & \multicolumn{2}{|c|}{$\begin{array}{c}\text { Ano de início } \\
\text { [2] }\end{array}$} & \multirow{2}{*}{$\begin{array}{c}\text { Avaliação } \\
\text { trienal } \\
2013\end{array}$} & \multirow{2}{*}{$\begin{array}{c}\text { Docentes } \\
\text { permanentes } \\
2010-12 \text { [3] }\end{array}$} \\
\hline & & & M & D & & \\
\hline UFRJ & Astronomia & $\begin{array}{l}\text { Astrofísica de } \\
\text { Altas Energias, } \\
\text { Extragaláctica } \\
\text { e Cosmologia }\end{array}$ & 2003 & 2010 & 4 & 20 \\
\hline UFRJ & Física & $\begin{array}{l}\text { Partículas } \\
\text { Elementares }\end{array}$ & 1972 & 1979 & 7 & 61 \\
\hline UFSC & Física & $\begin{array}{l}\text { Teoria de } \\
\text { Campos }\end{array}$ & 1988 & 1996 & 5 & 31 \\
\hline $\begin{array}{l}\text { Unesp/ } \\
\text { Guar. }\end{array}$ & Física & $\begin{array}{l}\text { Teoria de } \\
\text { Campos }\end{array}$ & 1990 & 1990 & 4 & 13 \\
\hline Unicamp & Física & $\begin{array}{l}\text { Astrofísica } \\
\text { de Neutrinos; } \\
\text { Fenomenologia } \\
\text { de Partículas; } \\
\text { Raios } \\
\text { Cósmicos }\end{array}$ & 1969 & 1969 & 7 & 80 \\
\hline Unifal & Física & $\begin{array}{l}\text { Exp. de Altas } \\
\text { Energias; } \\
\text { Fenomenologia } \\
\text { de Partículas }\end{array}$ & 2012 & .. & 3 & 17 \\
\hline Unifei & $\begin{array}{l}\text { Física e } \\
\text { matemática } \\
\text { aplicada }\end{array}$ & $\begin{array}{l}\text { Teoria de } \\
\text { Campos; } \\
\text { Gravitação e } \\
\text { Cosmologia }\end{array}$ & 2006 & .. & 3 & 20 \\
\hline USP & Física & $\begin{array}{l}\text { Partículas } \\
\text { Elementares e } \\
\text { Campos }\end{array}$ & 1970 & 1970 & 7 & 128 \\
\hline USP/SC & Física & $\begin{array}{l}\text { Teoria de } \\
\text { Campos; } \\
\text { Astrofísica de } \\
\text { Partículas } \\
\end{array}$ & 1975 & 1975 & 7 & 71 \\
\hline UFBA & Física & $\begin{array}{l}\text { Teoria de } \\
\text { Campos; } \\
\text { Gravitação e } \\
\text { Cosmologia }\end{array}$ & 1975 & 2007 & 3 & 38 \\
\hline UFPA & Física & $\begin{array}{l}\text { Partículas e } \\
\text { Campos }\end{array}$ & 2002 & 2010 & 4 & 17 \\
\hline
\end{tabular}

Fonte: Capes - Sistema Nacional de Pós-Graduação.

Legenda: [1] Mestrado profissionalizante. [2] Modalidade: M=mestrado; D=doutorado. [3] Média anual (docentes/ano) do programa.

No estudo dos 32 programas de pós-graduação em Física e Astronomia com linhas de pesquisa em $F A E$, foi comprovado que quase a 
totalidade das instituições responsáveis pela atividade científica na área são universidades, exceto o CBPF e o Instituto Nacional de Pesquisas Espaciais (INPE). Por outro lado, com exceção da PUC-Rio, todas as demais instituições pertencem ao setor público. A distribuição dos 32 PPGs em Física e Astronomia com linhas de pesquisa em FAE por região mostrou que o Sudeste tem 19 PPGs, o Nordeste seis, o Sul cinco, e o Centro-Oeste e o Norte um PPG cada um. Conforme observado, no desenvolvimento da pesquisa da FAE brasileira, destaca-se a região Sudeste, com protagonismo principal dos estados do Rio de Janeiro e de São Paulo, uma vez que ambos possuem 14 PPGs, pouco menos da metade do total. A partir do resultado da avaliação trienal 2013, 11 PPGs com linhas de pesquisa em FAE podem ser considerados com perfil internacional e de excelência, apresentando oito PPGs nota sete e três PPGs nota seis. Existem cinco PPGs com conceito cinco (nota máxima para programas com mestrado apenas) e 11 PPGs com nota quatro, constituídos majoritariamente por aqueles que iniciaram seus cursos de doutorado nos últimos anos. Por último, dos cinco programas com nota três, quatro deles são caracterizados pela recente abertura dos cursos de mestrado.

Na percepção de Chaves et al. (2007), embora o sistema de pósgraduação em Física tenha alcançado um nível aceitável, ainda não é capaz de formar pesquisadores experimentais na quantidade e qualidade suficientes por causa da falta de infraestrutura e de laboratórios mais modernos. Formam-se doutores na área sem contato com os instrumentos utilizados nos laboratórios internacionais. 0 autor entende que essa deficiência é minimizada por estágios no exterior com bolsas do tipo sanduíche. Waga et al. (2011) enfatizam a necessidade de alcançar um maior equilíbrio entre pesquisa teórica e experimental no país, pois hoje se pende mais para aquela do que para esta. Nesse sentido, a expansão do investimento em infraestrutura é fundamental para que o Brasil possa aumentar o número de pesquisadores experimentais e ampliar seu poder computacional na grande rede internacional de processamento de dados (GRID).

Com relação ao corpo docente, a comunidade brasileira da FAE contava, em 2012, com 532 professores, sendo que as subáreas de atuação se dividiam em: Teoria de Campos (37,5\%), Cosmologia e Gravitação 
$(17,8 \%)$, Fenomenologia (17,2\%), Experimental de Altas Energias (13,1\%) e o restante dividido entre Astronomia, Nuclear e outros (SBF, 2012). Na pesquisa realizada pela Sociedade Brasileira de Física (SBF), nota-se a existência de uma quantidade pequena de físicos experimentais. Apesar de a comunidade experimental se organizar por meio da Renafae, que também conta com a participação dos físicos teóricos, ainda falta uma maior conscientização sobre o desenvolvimento de instrumentação especializada e laboratórios nacionais a fim de possibilitar a ampliação do ensino e da pesquisa (SBF, 2012). Nesse sentido, a Capes (2013) recomenda aos programas existentes que priorizem a parte experimental na substituição ou ampliação do seu corpo docente com vistas ao desenvolvimento da área.

Ainda com as dificuldades apresentadas, a participação do Brasil nas colaborações internacionais da FAE aumentou significativamente e contribuiu para o aumento do número de publicações e citações da área nas bases de dados (SANTORO; NOVAES, 2003; CBPF, 2011). Os resultados apresentados na Tabela 1 confirmam o aumento dos artigos em colaboração e, especialmente, aqueles em colaboração internacional ao longo dos anos estudados.

A expansão dos programas de pós-graduação em Astronomia e Física e o consequente aumento do número de titulados em cursos de doutorado, mestrado acadêmico e mestrado profissional também são fatores a serem considerados na avaliação do crescimento de publicações nacionais em FAE. OCBPF foi a instituição pioneira na pesquisa experimental e teórica no Brasil, criando o primeiro PPG com cursos de mestrado e doutorado em 1962. Nos anos subsequentes, a atividade científica e a formação de recursos humanos se concentraram, principalmente, em universidades públicas da região Sudeste, estabelecendo-se nessa região mais quatro programas na década de 1960 e oito na década de 1970. Nos anos de 1980, foi criado apenas um programa com linha de pesquisa em FAE na região Sul, significando que a elaboração de indicadores de produção científica e o desenvolvimento de novos profissionais continuou em mãos da região Sudeste, como consequência do maior apoio financeiro dos órgãos locais de fomento à pesquisa. Na década de 1990, registrou-se um aumento considerável do número de programas (nove) em decorrência 
de ações promovidas por agências do governo federal, como CNPq, Capes e Finep (FAPESP, 2011). Esse indicador justifica o melhor ajuste ao modelo de regressão polinomial do período de 1993 a 2002 (Figura 2). Na década de 2000, o crescimento se manteve estável, com acréscimo de nove PPGs. Entretanto, houve uma ampliação do número de instituições participantes nas regiões Sul, Nordeste e Norte na pesquisa da FAE.

Na Figura 3, observa-se um crescimento contínuo tanto do número de publicações anuais quanto do número de grupos do CNPq e de PPGs na série temporal analisada. De fato, o aumento da produtividade da área pode ter sido consequência da expansão da pesquisa a partir da criação de novos PPGs e comunidades científicas. Da mesma forma, a ampliação do número de bolsas e incentivos, a maior cobertura das bases de dados internacionais e o incremento dos trabalhos em coautoria contribuíram para o desenvolvimento da disciplina no âmbito nacional.

\section{Figura 3 - Dinâmica de crescimento das publicações anuais, grupos do CNPq e PPGs da FAE brasileira (1983-2013)}
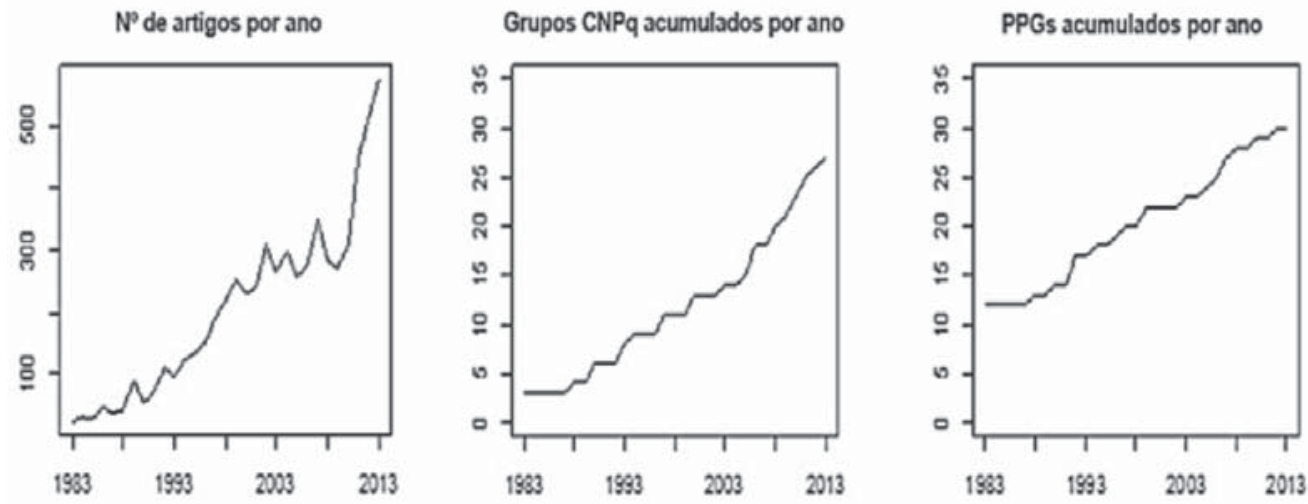

Fonte: Web of Science.

Percebe-se que a instituição da Renafae em 2008, por parte do $\mathrm{MCT}$, foi significativa para a área, visto que o número de publicações e grupos do CNPq aumentou consideravelmente a partir desse ano. Embora o crescimento do número de PPGs tenha sido menos acentuado, houve consolidação e ampliação dos programas com linhas de pesquisa em FAE. Segundo o CBPF (2011), a Renafae tem desempenhado um papel articulador fundamental para as colaborações brasileiras em programas internacionais, como os experimentos realizados no CERN (Suíça) e no Observatório Pierre Auger (Argentina). 


\section{CONSIDERAÇÕES FINAIS}

O desenvolvimento da Física de Altas Energias no Brasil entre 1983 e 2013 foi discutido a partir da análise da evolução da sua produção científica, dos grupos de pesquisa registrados do CNPq e programas de pós-graduação. A produção científica da FAE apresentou crescimento significativo ao longo da série temporal analisada, registrando uma média de crescimento anual de $14,27 \%$. Infere-se que a expansão da atividade da área pode ter sido consequência de vários fatores, dentre os quais: a ampliação do número de PPGs em Astronomia e Física com linhas de pesquisa em $F A E$, a ampliação do número de professores titulados e grupos de pesquisa do CNPq, a participação do Brasil nos projetos experimentais multinacionais e a criação da Renafae. Na década de 1990, verificou-se um aumento considerável do número de PPGs com linhas de pesquisa em FAE em virtude de ações promovidas por órgãos de fomento vinculados ao governo federal, como CNPq, Capes e Finep. A criação da Renafae, em 2008, foi um marco importante na área, pois o número de artigos publicados e grupos do CNPq aumentou consideravelmente a partir desse ano. A superioridade das publicações em colaboração com instituições estrangeiras demonstra a forte internacionalização da pesquisa em $F A E$, implicando um maior impacto da ciência brasileira no contexto global.

Os indicadores bibliométricos sugerem o amadurecimento da comunidade brasileira de FAE em decorrência da sua longa trajetória na pesquisa experimental. Sem dúvida, o status de país membro associado no CERN poderá aumentar o crédito do Brasil em projetos multinacionais em Big Science, consolidar áreas de expertisena instrumentação e ferramentas de análise de dados (softwares) e ampliar a influência da ciência realizada no país no cenário internacional. Espera-se que este estudo possa ter contribuído para a ampliação do conhecimento sobre a FAE brasileira e que consiga servir de instrumento para aperfeiçoar políticas e investimentos em diferentes âmbitos, como cursos de pós-graduação, infraestrutura nacional, colaboração internacional e inovação tecnológica.

\section{Agradecimentos}

Ao Conselho Nacional de Desenvolvimento Científico e Tecnológico (CNPq) pelo financiamento da pesquisa. 


\section{Referências}

ALMEIDA, E. C. E. de; GUIMARÃES, J. A. Brazil's growing production of scientific articles: how are we doing with review articles and other qualitative indicators? Scientometrics, Amsterdam, v. 97, n. 2, p. 287315, 2013.

CALERO, A. I. B. La colaboración y la visibilidad en las disciplinas de Física en Science Citation Index y arXiv (2000-2005). 2009. 476 f. Tese (Doutorado) - Universidad Carlos III de Madrid, Departamento de Biblioteconomía y Documentación, Getafe, 2009.

COORDENAÇÃO DE APERFEIÇOAMENTO DE PESSOAL DE NÍVEL SUPERIOR - CAPES. Documento de área 2013: Astronomia/Física. Brasília, DF: Capes, 2013.

CENTRO BRASILEIRO DE PESQUISAS FÍSICAS - CBPF. Plano Diretor do CBPF 2011-2015. Rio de Janeiro: CBPF, 2011.

CHAVES, A. et al. Relatório apresentado ao Ministério da Ciência e Tecnologia sobre alguns aspectos da Física brasileira. São Paulo: SBF, 2002.

Física para um Brasil competitivo. Brasília, DF: Capes, 2007. Disponível em: <https://goo.gl/Ou4IUv>. Acesso em: 30 mar. 2015.

COLLAZO-REYES, F.; LUNA-MORALES, M. E. Física mexicana de partículas elementales: organización, producción científica y crecimiento. Interciencia, Caracas, v. 27, n. 7, p. 347-353, 2002.

DIRETÓRIO DOS GRUPOS DE PESQUISA NO BRASIL LATTES - DGP. Base corrente. Brasília, DF: CNPq, 2014. Disponível em: <http://lattes.cnpq.br/ web/dgp>. Acesso em: 24 jan. 2015.

DUARTE, R. P. Cooperação Internacional para o Desenvolvimento em Ciência e Tecnologia: a participação brasileira na Organização Europeia para Pesquisa Nuclear (CERN). Journal of Technology Management \& Innovation, Santiago, v. 3, n. 4, p. 133-151, 2008. 
FUNDAÇÃO DE AMPARO À PESQUISA DO ESTADO DE SÃO PAULO - FAPESP. Análise da produção científica a partir de publicações em periódicos especializados. In: Indicadores de ciência, tecnologia e inovação em São Paulo - 2010. São Paulo: Fapesp, 2011. cap. 4.

GOLDEMBERG, J. 100 anos de física no Brasil. Rio de Janeiro: CBPF, 1973.

INSTITUTE OF PHYSICS. Bibliometric evaluation and international benchmarking of the UK's physics research. London: IOP, 2012. Disponivel em: <https://goo.gl/R02Dau>. Acesso em: 17 fev. 2015.

LÉPINE-SZILY, A. Relatório sobre o acordo Brasil-CERN. São Paulo: SBF, 2011. Disponivel em: <https://goo.gl/DKm50l>. Acesso em: 25 mar. 2015.

LETA, J. Brazilian growth in the mainstream science: the role of human resources and national journals. Journal of Scientometrics Research, Mumbai, v. 1, n. 1, p. 44-52, 2012.

MELE, S. et al. Quantitative analysis of the publishing landscape in highenergy physics. Journal of High Energy Physics, Trieste, v. 12, p. 1-25, 2006.

MOTOYAMA, S. À guisa de introdução: ciência e tecnologia no Brasil: para onde? In: . Prelúdio para uma história: ciência e tecnologia no Brasil. São Paulo: Edusp, 2004. p. 17-58.

SANTORO, A. F. S.; NOVAES, S. F. Física de altas energias: características e particularidades da área. Rio de Janeiro: SBF, 2003.

SOCIEDADE BRASILEIRA DE FÍSICA - SBF. A física no Brasil. São Paulo: SBF, 1987.

Relatório: a física e o desenvolvimento nacional. Brasília, DF: Centro de Gestão e Estudos Estratégicos, 2012.

SCHWARTZMAN, S. Um espaço para a ciência: a formação da comunidade científica no Brasil. Brasília, DF: MCT, 2001. 
SHELLARD, R. C. Física de altas energias no Brasil. Tlaxcala: Centro Brasileiro de Pesquisas Físicas, 2011.

SISTEMANACIONALDEPÓS-GRADUAÇÃO-SNPG. Cursos Recomendados/ Reconhecidos e Cadernos de Indicadores. Brasília: Capes, 2015. Disponível em: <http://www.capes.gov.br/avaliacao/dados-do-snpg>. Acesso em: 26 jan. 2015.

VANZ, S. A. de S. As redes de colaboração científica no Brasil: (20042006). 2009. 204 f. Tese (Doutorado) - Universidade Federal do Rio Grande do Sul, Faculdade de Biblioteconomia e Comunicação, Porto Alegre, 2009.

VANZ, S. A. de S.; STUMPF, I. R. C. Scientific output indicators and scientific collaboration network mapping in Brazil. Collnet Journal of Scientometrics and Information Management, Bangalore, v. 6, n. 2, p. 315-334, 2012.

VIEIRA, C. L.; VIDEIRA, A. A. P. História e historiografia da física no Brasil. Fênix - Revista de História e Registros Culturais, Rio de Janeiro, v. 4, n. 3, p. 1-27, jul./set., 2007.

. O papel das emulsões nucleares na institucionalização da pesquisa em física experimental no Brasil. Revista Brasileira de Ensino de Física, São Paulo, v. 33, n. 2, p. 2603-2611, 2011.

WAGA, I. et al. Física de partículas e campos. In: NOGUEIRA, S.; ROMERO, T. (Org.). Física 2011: estado da arte, desafios e perspectivas para os próximos cinco anos. São Paulo: Chris McHilliard, 2011. p. 155-174.

Recebido em 16/09/2016 Aprovado em 21/10/2016 